\title{
Estrategias neurocognitivas dirigidas al desarrollo del lenguaje químico en niños de primer grado
}

Neurocognitive strategies directed at development of chemical language in

first graders

\author{
Jonathan Alexander Jiménez Villegas \\ jonathanajv17@gmail.com \\ Código ORCID: 0000-0002-9740-0478 \\ Ministerio del Poder Popular para la Educación, Venezuela
}

\author{
Andrés Luciano Vivi Ramos
}

lucianovivi87@gmail.com

Código ORCID: 0000-0002-0545-8067

Ministerio del Poder Popular para la Educación, Venezuela

Recibido abril 2020 | Arbitrado mayo 2020 | Publicado junio 2020

\begin{abstract}
Resumen
Palabras clave:

Estrategias;

enseñanza; lenguaje;

neurocognición;

química

El presente trabajo de investigación tuvo por objetivo general implementar un programa de estrategias neurocognitivas para el desarrollo del lenguaje químico en niños de primer grado de la E.B. "Dr. Lisandro Lecuna"; se fundamentó en la teoría de los operadores constructivos y la neurociencia. Esta investigación se adscribió al enfoque cualitativo cuyo método fue la Investigación Acción Participante; los informantes clave fueron los estudiantes del primer grado de dicha institución. Como técnicas e instrumento se aplicaron la observación participativa y la entrevista; haciendo uso también de los registro anecdóticos. El desarrollo de la investigación se llevó a través de fases, fase I búsqueda de testimonios y aportes, fase II encuentros con los informantes, fase III ejecución del plan de acción y fase IV, comprendió procesos de reflexión permanente, durante el desarrollo de la investigación. Se concluyó que todas las estrategias elaboradas fueron medio experimental para que los estudiantes asociaran las palabras.
\end{abstract}

\section{Abstract}

Keywords:

Teaching; language; neurocognition, chemistry
The present research work had the general objective of implementing a program of neurocognitive strategies for the development of chemical language in first-grade children of the E.B. "Dr. Lisandro Lecuna "; it was based on theory of constructive operators and neuroscience. This research was assigned to the qualitative approach whose method was the Participating Action Research; the key informants were the first grade students of that institution. Participatory observation and interview were applied as techniques and instruments; also making use of anecdotal records. The development of the investigation was carried out through phases, phase I search for testimonies and contributions, phase II meetings with informants, phase III execution of the action plan and phase IV, included processes of permanent reflection, during the development of the investigation. It was concluded that all the strategies elaborated were experimental means for the students to associate the words. 


\section{INTRODUCCIÓN}

Ha sido un hecho muy conocido, el que a muchos estudiantes durante su etapa de educación media e incluso en la universidad, al enfrentarse al estudio de la química, unos más que otros encuentran dificultades de aprendizaje en general y en particular para ciertos temas de esta disciplina; principalmente se manifiestan en el bajo rendimiento académico, el poco interés por su estudio, la repitencia de la asignatura y usualmente una actitud muy poco positiva en el aula.

Entre las dificultades de origen externo se tienen la de origen económico y social, mientras de origen interno están todas aquellas relacionadas con la capacidad mental como es en el caso del trastorno de aprendizaje no verbal, la disortografía y la dislexia; alteraciones que afectan la motivación de muchos estudiantes; y por otro lado todas aquellas que puedan estar vinculadas con la demanda de una tarea, bien sea todas aquellas derivadas de la preparación académica previa a los estudiantes y las derivadas de la naturaleza propia de la química.

Haciendo uso de los planteamientos de Pascual-Leone et al. (1970), es posible buscar explicaciones para las dificultades que se presentan en algunos estudiantes a la hora de adquirir y dominar nuevas habilidades y conocimientos entorno a los temas de química. Pascual-Leone en procura de hacer más funcional la teoría de Piaget, formuló la teoría Neo-Piagetiana la cual ha sido modificada y ampliada, por el mismo y sus colaboradores con la intensión particular de determinar cuantitativamente un parámetro que permita explicar el funcionamiento del mecanismo central de atención o memoria de trabajo, el cual llamo operador M; por ende el comportamiento está determinado por una unidad estructural a la que denominó esquema el cual se va activando mediante estímulos representados en un principio por procesos afectivos como la motivación, el autoestima y la seguridad que en un segundo plano lleva a la necesidad de asociar saberes previos con nuevos fenómenos percibidos $\mathrm{u}$ observados que finalizan en un aprendizaje lógico-estructural automatizado derivado del sobre-aprendizaje, con lo que un esquema de conocimientos previos es absolutamente necesario para la adaptación de nuevas competencias educativas y determinan en gran parte el éxito o fracaso de un sujeto al encarar una tarea cognitiva.

Por consiguiente, el origen de estas dificultades de estos estudiantes se les puede diagnosticar como una estructura cognitiva incorrecta a la hora de procesar y agrupar la información entre un contexto real que se relacione con fenómenos de la vida cotidiana y un contexto conceptual propio de la química. Pues esta es una asignatura que en nuestro país se empieza a impartir en un noveno grado de bachillerato, por lo que cada uno de los estudiantes se encuentra en la etapa de la adolescencia que en consecuencia de sus peculiares confusiones y elecciones importantes que conllevan sus recientes cambios hormonales, están desorientados y como no manejan conocimientos previos de química sienten que no pueden lidiar con muchos de los conceptos básicos de la química y por lo tanto no pueden resolver de manera eficiente los problemarios y actividades que se les asignan entorno a sus obligaciones. 
Todo docente sin excepción los del área de la química debe tener en cuenta que, la demanda de una tarea o de una pregunta en medio de la clase, requiere del mayor número de pasos o de esquemas de pensamiento que el estudiante debe emplear para poder resolverla con éxito. Por lo que es esencial un excelente dominio de los contenidos impartidos en clases posteriores por parte de los estudiantes. Por esta razón Nakamatsu (2012) menciona:

El aprendizaje de la química es difícil, pues requiere que el estudiante sea capaz de relacionar el mundo macroscópico que percibe con un mundo submicroscopico basado en átomos y moléculas que no puede percibir, y debe, además, poder aprender un sistema de símbolos necesarios para su representación. (p. 38)

Esto quiere decir, para cada persona el entendimiento de una clase de química es diferente y varia con los conocimientos previos, que de no tenerlo dificulta mucho el aprendizaje, ocasionando una sobrecarga de la memoria de trabajo por la cantidad de información que esta debe manejar en poco tiempo, competencia que muchos estudiantes no pueden lograr dificultando la tarea del docente, dando la impresión de que la materia es muy complicada, ocasionando una alta deserción estudiantil en carreras universitarias relacionadas con el campo de la química.

Tomando como referencia lo dicho por la pediatra y neuropediatra española Mas (2015) en su blog electrónico llamado Neuronas en crecimiento basándose en el criterio de muchos estudios científicos establece:

Durante la niñez después de
cumplido los tres años de vida
culmina la etapa del dominio del
habla y en los siguientes siete
años comienza la etapa del
neurodesarrollo del lenguaje en
donde adquirimos el dominio del
lenguaje y con él la evolución del
pensamiento. (parr.22)

Con lo que podría decirse, una posible solución a este gravísimo problema sería la incorporación temprana y consistente del lenguaje químico en niños de Educación Primaria. Afirmando que el periodo más propicio para la adquisición de habilidades y destrezas viene comprendido entre los primeros 4 y 10 años de vida ya que en esta etapa particular de la niñez humana el cerebro presenta una alta disposición neuronal, por lo que es un periodo muy sensitivo que le permite al niño retener información con mucho menor esfuerzo que un adulto o un adolescente.

El lenguaje químico tanto para la ciencia como para la educación se emplea para facilitar la comunicación de procesos y circunstancias que explican de manera exacta un fenómeno, estrechamente ligado a la química, logrando de esta manera un nivel de formalidad tanto en el habla como en la escritura mediante el uso de concepciones, palabras y símbolos que constantemente se traducen de un lenguaje verbal a uno simbólico o viceversa, por lo tanto la función del profesor es enseñar a los alumnos el razonamiento discursivo sobre los procesos, basándose en la teoría para entender porque se realiza tal o cual 
procedimiento en el laboratorio, que según el juicio de Roaux (2006):

(...) la importancia del lenguaje en el aprendizaje de la química radica en reconocer que docentes y alumnos deben compartir los conocimientos empleando un lenguaje que debe ser unívoco, de forma que no se transforme en un obstáculo al momento en que el alumno construya nuevos marcos teóricos. (p.17)

Por lo que se puede concluir que el dominio de la asignatura depende del dominio de su lenguaje. A su vez, Facundo Manes (2014) medico neurólogo y neurocientífico argentino creador y director de INECO (Instituto de Neurología Cognitiva) sostiene:

La educación se va a beneficiar del estudio y los avances del cerebro, porque si el docente entiende como el cerebro del alumno teoriza, jerarquiza, toma decisiones, memoriza y olvida; el docente puede transmitir mejor el mensaje, sea el mensaje A, B o C con la técnica de enseñanza $A, B$ o $C$. (En línea)

Así que hoy en día la neurociencia nos ofrece una nueva gama de conocimientos acerca del cerebro que pueden ser muy útiles a la hora de diseñar estrategias educativas enfocadas en los mecanismos de atención, codificación, consolidación y evocación, los cuales son indispensables para la reconstrucción y manipulación de la memoria. Por otro lado, en lo que respecta al desarrollo intelectual Manes (ob. cit.) señala bajo los nuevos avances de la neurociencia:
El aprendizaje se da en sociedad y con el contacto humano, cuando algo nos motiva y nos parece un ejemplo, lo cierto es que desde pequeños estamos aprendiendo permanentemente, por lo que el aprendizaje es tan central en la vida que es imprescindible tratar de comprender sobre cómo se produce en nuestro cerebro el aprendizaje y como se pueden mejorar estos procesos en lo individual pero también en lo social. (En línea)

Por último, es conveniente acotar la relación ya existente entre neurociencia y educación que en conjunto con lo antes mencionado la educadora y neurocientifica Willis (2007) pone a la disposición dos puntos relevantes que deben ser considerados por todo docente a la hora de elaborar estrategias didácticas que conlleven a un óptimo aprendizaje, los cuales describió de la siguiente manera:

En primera instancia tenemos el estado de ánimo del alumno o la predisposición que este tenga hacia la captación de una información novedosa. Si el alumno está contento, la información recepcionada será aprendida con mayor facilidad, en situación contraria de nada valdrán las explicaciones del profesor aun cuando destilen calidad. En segundo lugar, está la metodología empleada en la enseñanza porque depende en gran parte de la manera como el estudiante se predisponga para aprender. (p.125)

Ahora bien, por todo lo anteriormente señalado, surge como interrogante en este 
estudio, ¿Qué estrategias neurocognitivas serían propicias para el desarrollo del lenguaje químico en niños de primer grado enfocado en las propiedades intensivas y extensivas de la materia? Para dar respuesta a la misma, se hizo necesario plantearse como objetivo general implementar un programa de estrategias neurocognitivas para el desarrollo del lenguaje químico en niños de primer grado de la Escuela Básica "Doctor Lisandro Lecuna".

Como objetivos específicos, se plantearon en primer lugar identificar el proceso de enseñanza y aprendizaje de las ciencias naturales y el desarrollo del lenguaje químico desde la realidad escolar. Seguidamente, diseñar un programa de estrategias neurocognitivas para la comprensión del lenguaje químico desde las ciencias naturales en los estudiantes de primer grado de Educación Primaria partiendo de su realidad. Luego, ejecutar las estrategias neurocognitivas durante el proceso enseñanza y aprendizaje de las ciencias naturales para el desarrollo del lenguaje químico mediante la comprensión de las propiedades extensivas e intensivas de la materia. Para así finalmente analizar los cambios surgidos en el lenguaje de los niños de primer grado de la Escuela Básica "Dr. Lisandro Lecuna" tras el desarrollo del programa.

Vale destacar, el motivo por el cual se realizó la presente investigación surgió de la necesidad que tiene la educación venezolana de redimensionar el rol del docente mediador en las aulas de clase; ya que este no cuenta con las capacidades necesarias para el desarrollo eficaz y eficiente del acto pedagógico, evidenciado en la no utilización de estrategias innovadoras para impartir la clase, convirtiéndose en una educación tradicional, tal caso es con las ciencias naturales haciendo énfasis en la química la cual es conocida como una ciencia de difícil comprensión, basada en fórmulas $\mathrm{y}$ reacciones químicas, tornándose pesada para los discentes, lo que conlleva a una alta deserción escolar; es por esto que, resulta de suma importancia implementar un programa de estrategias neurocognitivas para el desarrollo del lenguaje químico en niños de primer grado con el fin de que conozcan más sobre esta ciencia y se relacionen con el gran mundo microscópico y macroscópico que los rodea, es necesario incentivar a los niños a comprender la química y así desarrollar un conocimiento científico desde pequeños, estimulando diferentes partes del cerebro, logrando un lenguaje correcto.

Es por ello, que las estrategias empleadas durante el proceso enseñanza y aprendizaje de las ciencias naturales para el desarrollo del lenguaje químico parten de un tema fundamental siendo este sobre las propiedades extensivas e intensivas de la materia, que son aquellas que dependen de la extensión de esta; es decir, dependen de la cantidad de materia $u$ otras propiedades relacionadas con la cantidad de materia, por ejemplo el volumen, con una relación proporcional entre el cambio en la cantidad de materia y el cambio en las propiedades extensivas que provoca, en el mismo orden de ideas las propiedades intensivas. Por el contrario, son aquellas propiedades que son independientes de la cantidad de materia, cabe destacar que este tema no se debe dar con mucha complejidad y que la intención es desarrollar un lenguaje científico mediante estrategias neurocognitivas en niños de 
primer grado de la E.B. "Dr. Lisandro Lecuna" tras el desarrollo del programa. El estudio buscó atender las necesidades presentes en la línea de investigación Pedagogía, Andragogía y Gerencia aplicada a la Biología y Química, correspondiente al Departamento de Biología y Química de la Facultad de Ciencias de la Educación Universidad de Carabobo.

\section{MÉTODO}

La presente investigación se adscribió a un paradigma interpretativo con enfoque cualitativo, de acuerdo con Taylor y Bogdan (1986) los cuales consideran, en un sentido amplio, la investigación cualitativa como "aquella que produce datos descriptivos: las propias palabras de las personas, habladas o escritas, y la conducta observable". (p.20); es decir, está basada en resolver problemas desde una perspectiva naturalista, fenomenológico, etnográfica, interpretativa donde las personas son los principales protagonistas, tomando en cuenta la comprensión de las complejas interrelaciones que se dan en la realidad.

En cuanto al método asumido por los investigadores es el de la Investigación Acción Participante (IAP) la cual es una metodología que apunta a la producción de un conocimiento propositivo $y$ transformador, mediante un proceso de debate, reflexión y construcción colectiva de saberes entre los diferentes actores de un territorio con el fin de lograr la transformación social.

Por lo tanto, se puede decir que la investigación acción participativa es un proceso dialéctico continuo en el que parte de que las personas viven en un contexto, crean cultura que se reproduce en sus dichos y hechos, se conceptualizan los problemas, se planifican y se ejecutan las acciones en busca de la transformación de los contextos, así como a los sujetos que hacen parte de la misma. Mientras el procedimiento asumido tras la revisión de múltiples textos alusivos a la práctica metodológica de la acción participativa, con el propósito de hallar la más propicia para la aplicación del proyecto, resultó por decisión unánime la establecida por Eizagirre y Zabala (2006). autores del diccionario de acción humanitaria y cooperación al desarrollo, siendo esta obra el medio por el que explican y ratifican:

El método de la investigaciónacción participación (IAP) combina dos procesos, el de conocer y el de actuar, implicando en ambos a la población cuya realidad se aborda. Al igual que otros enfoques participativos, la IAP proporciona a las comunidades y a las agencias de desarrollo un método para analizar y comprender mejor la realidad de la población (sus problemas, necesidades, capacidades, recursos), y les permite planificar acciones $\mathrm{y}$ medidas para transformarla $y$ mejorarla. Es un proceso que combina la teoría y la praxis, y que posibilita el aprendizaje, la toma de conciencia crítica de la población sobre su realidad, su empoderamiento, el refuerzo y ampliación de sus redes sociales, su movilización colectiva y su acción transformadora. (p.1)

Esto significa que la IAP busca transformar la realidad, convertir las 
debilidades en fortalezas, atendiendo a las necesidades existentes en la realidad educativa. De hecho, para seguir estos procesos Colmenares (2012) en su artículo Investigación-Acción Participativa: una metodología del conocimiento y la acción propuso un programa de actividades afirmando lo siguiente:

Como seguidora de esta metodología, en mis desarrollos investigativos presento cuatro fases, a saber: Fase I, descubrir la temática; Fase II, representada por la construcción del Plan de Acción por seguir en la investigación; la Fase III consiste en la Ejecución del Plan de Acción, y la Fase IV, cierre de la Investigación, en la cual se sistematizan, categorizan y generan aproximaciones teóricas que pueden servir de orientación para nuevos ciclos de la investigación, creando un binomio entre el conocimiento y la acción, procesos que coadyuvan a la potenciación de las transformaciones esperadas; por supuesto que todas estas fases van integradas por procesos reflexivos permanentes de todos los investigadores involucrados.(p.6)

En función a lo anterior, para el desarrollo de este estudio se asumió como Fase I, la relacionada con descubrir una preocupación temática, se puede llevar a cabo con la búsqueda de testimonios, aportes y consideraciones de los investigadores interesados en la misma. Como Fase II, conllevó a realizar algunos encuentros con los interesados, a fin de delinear las acciones acordadas por consenso que el grupo considere más acertadas para la solución de la situación identificada o los problemas existentes en un área de conocimiento, en una comunidad, una organización, en fin, en una realidad seleccionada. El escenario tuvo lugar en la Escuela Básica "Dr. Lisandro Lecuna", ubicado en Naguanagua, Estado Carabobo en el periodo 2018-2019. Los informantes clave fueron los estudiantes de primer grado de dicha institución.

Mientras la Fase III, se corresponde con la ejecución del plan de acción que previamente se ha construido $y$ que representa las acciones tendientes a lograr las mejoras, las transformaciones o los cambios que se consideren pertinentes. $Y$ la Fase IV comprende procesos de reflexión permanente, durante el desarrollo de la investigación, además de la sistematización, codificación, categorización de la información, y la respectiva consolidación del informe de investigación que da cuenta de las acciones, reflexiones $y$ transformaciones propiciadas a lo largo de la investigación.

En cuanto a las técnicas para la obtención de información según Arias (2006) las define "como el conjunto de procedimientos y métodos que se utilizan durante el proceso de investigación, con el propósito de conseguir la información pertinente a los objetivos formulados en una investigación". (p.376). Por esta razón se empleó la técnica de observación participativa en donde el investigador se mezcla con la situación observada y coopera con las actividades al detectar los aspectos sociales, la observación juega un papel fundamental, para Taylor y Bogdan (2000) explican: 
En la observación será necesario tener en cuenta dos aspectos importantes que pueden influir en el resultado obtenido tras la misma, es el ocultamiento y la intervención. En el ocultamiento el individuo observado puede percatarse de la presencia del observador y distorsionar la conducta. La intervención denota el grado en que el investigador, a diferencia de un observador pasivo, estructura el ámbito de observación en respuesta a las necesidades del estudio. (p.60)

Al igual que la observación participante, también se empleó como técnica adicional de recolección de datos la entrevista, que según Kerlinger (1985) puede entenderse como "una confrontación interpersonal, en la cual una persona formula a otra preguntas cuyo fin es conseguir contestaciones relacionadas con el problema de investigación" (p.338). Con la finalidad de que sea flexible y adaptable a los objetivos de la investigación se escogió un tipo particular de la misma, la entrevista semiestructurada.

En cuanto a la credibilidad y fiabilidad del instrumento, en el momento de presentar los resultados de una investigación cualitativa se deben tomar en cuenta tres criterios que permiten evaluar la calidad científica del proyecto desde la visión del valor de verdad, su aplicabilidad, la consistencia y la neutralidad. A estos tres criterios se les conoce como credibilidad, transferibilidad y comprobabilidad.

\section{RESULTADOS Y DISCUSIÓN}

Desde la perspectiva de la acción participativa como procedimiento metodológico para llevar a cabo nuestro proyecto transformador de la educación básica, se requiere combinar dos procesos, el conocer y el actuar para luego aplicar cada uno de ellos en la población cuya realidad se procedió a estudiar. En tal medida, siguiendo este orden de ideas en este apartado en primer lugar se pone en manifiesto la Fase I realizada durante la primera etapa del proyecto, destinada a conocer la realidad escolar que viven los estudiantes cursantes del 1er grado de la sección "A" de la Escuela Básica "Dr. Lisandro Lecuna".

La fase I se encuentra correspondida con la observación de la actividad en el aula y la entrevista a la docente del aula, para ello se apuntó cada uno de los aspectos más relevantes en un registro anecdótico. Lo primero a tener en cuenta es el hecho de que, el estudio de la realidad escolar representada por los niños de primer grado fue realizado durante la finalización de una jornada de actividades correspondiente a la rutina diaria de clases. Una vez en el aula fue muy evidente la experiencia de la docente en el ámbito de la planificación y realización de actividades con niños, pudiendo corroborar su relato acerca de la importancia atribuida a su viejo trabajo en el preescolar, que le permitió desarrollar una fuerte vocación por su carrera y desempeñarse de la mejor manera posible en su labor de mejorar las destrezas y conocimientos que traen los niños 
pequeños de sus hogares como también de niveles previos.

Entre la selección de estrategias didácticas llamó la atención el que, la profesora utilizara técnicas para la estimulación cognitiva como una actividad especial para ayudar a los niños con su desempeño en las actividades diarias teniendo un efecto positivo con el comportamiento y el aprendizaje. En cuanto a los métodos de enseñanza y aprendizaje ejercidos por la docente se encuentran el método activo caracterizado por la autogestión del aprendizaje por parte de sus alumnos el cual involucra una relación directa con la atención siendo esta una cualidad importante de la conducta, aunque quedo evidenciado que para poder captar la misma es necesaria una recopilación constante de las normas de comportamiento y lograr de esta manera un ambiente de disciplina, debido a la mala relación que tiene esta frente a los agentes distractores determinados por estímulos visuales y verbales que afectan de forma negativa el comportamiento de los chicos durante una clase.

Mientras por otro lado también se manifestó otro método de enseñanza vinculado a la autogestión del aprendizaje caracterizado por la inducción al razonamiento de parte de sus pequeños estudiantes, por lo que, la profesora busca con la utilización de ambos métodos incorporar en el aprendizaje de los pequeños, sus necesidades e intereses, los conocimientos previos emergentes de la interacción con su familia y su comunidad y un conocimiento obtenido por descubrimiento y de forma significativa.
Con respecto a la elaboración del proyecto de aprendizaje se comienza por un diagnostico estudiantil, que en primer lugar toma en cuenta el grupo etario, que se encuentra comprendido por niños con edades entre los 5 y 6 años que constituyen a su vez una matrícula de 35 alumnos inscritos que se ha visto afectada por el creciente problema de la deserción escolar ocasionada por factores socioeconómicos que limitan el apoyo de los representantes, dando origen a una asistencia regular de solo 25 alumnos, que además muchos de ellos por la misma problemática se encuentran perjudicados por una mala nutrición que los llevan a tener problemas en el aprendizaje por el terrible efecto que esta tiene sobre los procesos de atención derivados de la conducta, cuestión que representa un gran reto para los chicos en el instante que les toca demostrar los niveles de logro que han ido adquiriendo en el presente año escolar, lo que puede explicar un posible motivo por el cual se observó un bajo desempeño en las actividades por parte de un grupo importante de niños que exteriorizo dificultades para decodificar y reconocer palabras, poca habilidad para inferir en la lectura y en operaciones concretas además de poseer escasa velocidad y fluidez en la lectura.

Aunque de manera positiva se ha podido demostrar que estas dificultades mostradas por estos niños se pueden ir corrigiendo mediante la aplicación de sistemas de representación sensorial como principal estrategia de aprendizaje, siendo este uno de los aspectos considerados en la planificación de los objetivos del 
proyecto de aprendizaje, el cual busca satisfacer un modelo incluyente de educación basado en la necesidad estudiantil tanto de origen interno como externo.

Entre las necesidades de origen interno se evidenció aparte de las mencionadas en el párrafo anterior refiriéndose a los niveles de logro alcanzados por este grupo de estudiantes, se tienen además incluidas en él un caso de dislexia y otro de autismo, los cuales requieren de fundamentos metodológicos que les ayude a integrarse junto con los demás a la clase fomentando su participación y progreso en las asignaturas, por tanto, se requieren de didácticas integradoras que dirijan su atención al niño de forma tanto colectiva como individual.

Para ello la profesora señaló que en esta etapa los niños todavía se encuentran enraizados en lo que fue su trabajo en el preescolar trayendo esos hábitos consigo que les hace sentir ese apego hacia la mama y hacia los juegos, así que el desarrollo de sus clases se lleva mediante la ejecución de juegos que tienen instrucciones con lenguaje sencillo que van siguiendo una matriz integradora de las áreas de aprendizaje que de apoco le va creando experiencia a los chicos para que se vayan adaptando al sistema educativo, competencia que mantiene una relación provechosa con las actitudes manifestada por esto chicos cada día, mientras se realizaban las actividades correspondientes a la rutina diaria de clases en donde destacan valores como el compañerismo, el compromiso, la iniciativa, la convivencia y la adaptabilidad para los proyectos, atributos importantes que conducen a este maravilloso grupo a tener afinidad estudiantil, la cual es una de las fortalezas halladas en esta sección de estudiantes, sumando de esta manera un punto de vista positivo relacionado con el comportamiento que pueden llegar a tener.

Desde luego que estos hábitos que traen del preescolar también se encuentran asociados a los problemas de disciplina mencionados anteriormente, los cuales se reflejan en el mal comportamiento y el no acatar el cumplimiento de las normas de convivencia que pueden entorpecer la realización de una clase, situación que la profesora domina por medio del control verbal, por eso de vez en cuando se observó que algunos niños sostenían un lenguaje inadecuado con sus compañeros, entonces se busca corregir esta conducta con la autogestión de normas originadas de uno de los fundamentos metodológicos utilizados por la profesora basado en la teoría de la acción comunicativa de Habermas en donde todos los participantes son considerados iguales incluyendo a los chicos con autismo y dislexia respectivamente, por lo que su opinión es tan válida como la de la profesora o la de cualquier otro alumno.

Estas opiniones proceden de argumentos válidos que permitan llegar a un consenso favorable para todos, haciendo de este un año escolar prospero pese a los inconvenientes mencionados con anterioridad. Por otra parte, también se ve inmiscuida el apoyo de los representantes dando por hecho que se toma en cuenta a la familia y a la comunidad evidenciándose otro de los fundamentos metodológicos en el que se apoya la profesora del aula conocida como la teoría del aprendizaje socio-cultural de Vygotsky, que al ser los chicos participe en la creación de las normas de convivencia como en cada una de 
las etapas del proyecto de aprendizaje se aumentan las posibilidades de adquirir un aprendizaje significativo fundamentado por David Ausubel.

\section{CONCLUSIONES}

Como conclusión de esta investigación acción participante, se tiene que los niños han demostrado mucho interés por aprender y participar en todas las actividades escolares, completando con éxito las competencias impuestas de lectura y calculo, todo a un nivel inicial como se esperaba, es decir con ciertas deficiencias, pero se puede afirmar que saben leer, contar, sumar y restar.

Es por ello, para el logro de los objetivos planteados se procedió a la elaboración y descripción de las estrategias donde se planificó una clase sobre las propiedades no características de la materia y para ello se llegó a una decisión unánime de elaborar como estrategia de inicio un experimento de los sentidos al que se llamó "adivina y aprende". Para este experimento se pretendía preparar dos estaciones, en la primera se coloca una mesa y se decora de manera alusiva a las propiedades físicas de la materia utilizando juguetes y recipientes de uso cotidiano que contengan jugo de mandarina, jugo de mango, café molido y alcohol, del mismo modo se decorara otra mesa, pero esta vez alusiva a las propiedades químicas de la materia utilizando un cilindro de vidrio, frutas, botones oxidados, limón, huevos, agua y bicarbonato de sodio.

Todo esto con la intención de presentárselo a los niños en forma de juego, para ello se les convence que ese día todos serán químicos y como químicos el trabajo es estudiar la materia la cual es todo lo que rodea, inclusive uno mismo; mediante la utilización de una técnica de animación infantil, luego con la colaboración de la profesora a cargo se buscó armar grupos de seis niños para luego ir pasando cada grupo por cada una de las estaciones, durante el paso de la primera estación se le vendaron los ojos a cada estudiante y con la ayuda de un gotero se les dio a probar a cada uno varios jugos y con el sabor, ellos tenían que identificar de qué es el jugo; al terminar se les procederá a pasar cerca de la nariz un frasco con café molido y otro con alcohol.

Posteriormente identificaron cuál es cual con su olor, seguidamente se quitaron las vendas y compararon varios juguetes por su color tamaño y material para después pasar a la segunda estación en donde se les dará a palpar botones nuevos y botones oxidados, observaran el estado de descomposición de un cambur así como también la diferencia que hay entre un huevo crudo y otro cocido, de igual modo se les presentara una demostración de la reacción química entre el bicarbonato, el agua y el limón.

Esta dinámica se realizó con la finalidad de estimular las gnosis en los chicos para facilitarle el aprendizaje de las propiedades físicas y químicas de la materia mediante el uso de sus cincos sentidos. Para el momento del desarrollo de la clase se procedió a explicarles a los jóvenes la masa, el volumen y la temperatura mediante tres estrategias sencillas, en vista de que el objetivo de este encuentro es el manejo de las palabras químicas, materia, propiedades físicas, 
propiedades químicas, masa, volumen y temperatura, las exposiciones verbales se llevaran a cabo muy brevemente con palabras sencillas mediante técnicas de animación infantil acompañadas de breves dinámicas demostrativas.

Para el caso de la masa se utilizará una balanza digital para realizar un juego al que llamaremos descubriendo nuestra masa, en él se mostró el instrumento y se le dio uso pasando a cada niño de manera voluntaria y organizada a montarse en la balanza y memorizar su masa para luego comparar que estudiante tiene más masa y quien tiene menos. Esta dinámica se hace con la finalidad de que los niños aprendan de manera interactiva la importancia y el uso de la masa como magnitud física y estimular la memoria de trabajo.

Para el caso del volumen se realizó una dinámica en la que se tomaron cada uno de los juguetes utilizados en la estación de las propiedades físicas y se fueron colocando en una caja, para luego irle preguntando a cada niño según su propio criterio cual juguete ocupa más espacio dentro de la caja y cual ocupa menos. La finalidad de esta dinámica fue demostrar de manera interactiva la propiedad del volumen y estimular el mecanismo de la memoria de trabajo en los chicos facilitando su aprendizaje.

En términos generales haber realizado esta investigación ha dejado una enorme satisfacción pues los niños cursantes del primer grado de la sección "A" de la E.B. "Dr. Lisandro Lecuna" han demostrado ser un grupo muy motivado a aprender teniendo una buena afinidad estudiantil como principal característica, por otro lado, la profesora del aula se mostró muy colaborativa con nosotros al momento de realizar las actividades, que de no ser por su ayuda quizás, no se hubieran podido llevar a cabo como se esperaba. Factores que intervinieron directamente de manera muy positiva en los resultados de la presente investigación.

Durante la fase de observación fue evidente que la profesora del aula sigue el modelo incluyente de educación en su salón de clase, debido a que tiene un chico con dislexia y otro con autismo por lo que constantemente siempre está buscando adaptar su plan de clase a las necesidades de cada uno de sus estudiantes esto dos casos especiales y es aquí en este punto que las estrategias neurocognitivas para el desarrollo del lenguaje químico diseñada para esta investigación acción caen como anillo al dedo a este grupo de niños. Al terminar dichas actividades se llegó a la conclusión en que todas las estrategias neurocognitivas elaboradas realmente a nivel pedagógico cumplieron con su propósito; de hecho, el juego adivina $y$ aprende por ejemplo sirvió como medio experimental para que los niños asociaran las palabras olor, sabor, color, tamaño y textura con las propiedades físicas de la materia mediante a analogía de la experiencia vivida con las palabras escuchadas, igual ocurrió con las propiedades químicas de la materia y los demás juegos interactivos realizados ya sea en el descubriendo nuestra masa, la demostración de la caja para el volumen y el moléculas en acción todos realizados cada uno de los encuentros didácticos. Lo dicho se pudo corroborar en ese mismo instante con la dinámica de cierre el memo-química en la cual los participantes estaban 
motivados $y$ de hecho fue resuelta fácilmente por la gran mayoría de los niños sin ningún problema e inclusive antes de iniciar las clase el siguiente encuentro algunos estudiantes manifestaron seguir practicando en sus casas, hecho que tomó por sorpresa, dejando así en evidencia que los juegos inciden en el aprendizaje.

\section{REFERENCIAS}

Arias, F. (2006). El proyecto de Investigación. Introducción a la metodología científica. Venezuela: Episteme

Colmenares, A. (2012). Investigación-Acción Participativa: una metodología del conocimiento y la acción. Recuperado: https://revistas.uniandes.edu.co/doi

Eizagirre, M. y Zabala, N. (2006). Investigación-acción-participación (IAP). Recuperado:

https://icualitativa.wordpress.com/te mas/tema-2/investigacion-accionparticipacion-iap-marlen-eizagirre-ynestor-zabala/

Kerlinger, F. (1985). Investigación del comportamiento. Recuperado: https://es.scribd.com/document/3753 81810

Manes, F. (2014). Usar el cerebro, conocer nuestra mente para vivir mejor. Buenos Aires: Editorial Planeta
Mas, J. (2015). Neuronas en crecimiento. Recuperado:https://neuropediatra.org

Nakamatsu, J. (2012). Experiencias docentes. Reflexiones sobre la enseñanza de la química. Recuperado: http://revistas.pucp.edu.pe/index.php /

Ortiz, T. (2009). Neurociencia y educación. Alianza Editorial

Pascual-Leone A., Fernández, A. y Bartrés, D. (2019). El cerebro que cura. Plataforma Editorial

Pozo, T. y Gutiérrez, J. (2006). La triangulación analítica como recurso para la validación de estudios de encuesta. Recuperado:

https://www.redalyc.org/pdf/916/916 12206.pdf

Roaux, R. (2006). Una valoración de la comprensión lectora en alumnos del primer año de universidad, Educación Química., 17(1), 77-81

Rodríguez, G., Gil, J. y García, E. (2009) Metodología de la investigación cualitativa. Ediciones Aljibe. Granada

Taylor, S. y Bogdan, R. (2000). Introducción a los métodos cualitativos de investigación. México: Paidós

Willis, J. (2007). Neurociencia aplicada al aprendizaje y la educación. Recuperado: http://www.redem.org/boletin/boleti n300410b.php 